\title{
IDENTIFYING SIGNIFICANT FACTORS AFFECTING REQUEST FOR INFORMATION (RFI) PROCESS TIME
}

\author{
Chang-sun Chin \\ Jeffrey S. Russell \\ Department of Civil and Environmental Engineering \\ University of Wisconsin, Madison \\ Madison, WI 53706, USA
}

\begin{abstract}
The research is to illustrate how to identify the significant factor(s) affecting the Request For Information (RFI) process cycle time in the context of queueing behavior. Among three different factors involved in the general queueing model (i.e. variability factor, utilization factor, and average process time), the variability factor was selected and investigated to see how variations affect the process cycle time under different conditions along with the batching effect. In order to determine the levels of factors, a set of RFI data was statistically analyzed. Based on the selected factors with levels determined, different scenarios were developed and simulation study was conducted so as to see how the system performs differently. Then, results of simulation were analyzed using the DOE in order to identify the most significant factor(s) which affects the current process cycle time.
\end{abstract}

\section{INTRODUCTION}

As project complexity increases, the likelihood of problems - errors, conflicts, omission, and ambiguities - also increases (Zack 1999). The higher probability of problems exists the more risks likely occur. In construction industry, one of the important tools to reduce risks is the Request For Information (RFI) which is a vehicle for communication and clarification between owner and contractor. A delay of responses from the reviewer (design team) causes the contractor's delay, possibly resulting in the project delay. We could observe that each RFI has its own due date and the due dates are usually set forth by the contractor (i.e. contractor wants to receive responses within a certain period of time). However, owing to the reviewer's limited capacity of processing RFI, actual cycle times tend to be longer than the time the contractor wants to receive the responses.

\section{PRELIMINARY DATA ANALYSIS}

The first author conducted observations on RFI processing from several projects in different regions in the United
States and noted that the on-time rate of RFI process was very low on average (about 50\%) and the average response time (lead time) was considerably longer than the contractors typically expected. The individual project selected for this research involves an eight-story college laboratory building located in California in the United States. The data set for the research consists of 574 RFIs generated about seven month period, averaging a daily rate of about three RFIs. It is interesting to note that the dates when the contractor expected to receive the responses from the designer fell mostly into two time periods -7 days or 14 days (56\% and $28 \%$ respectively). Figure 1 represents the range of varying times expected by the contractor.

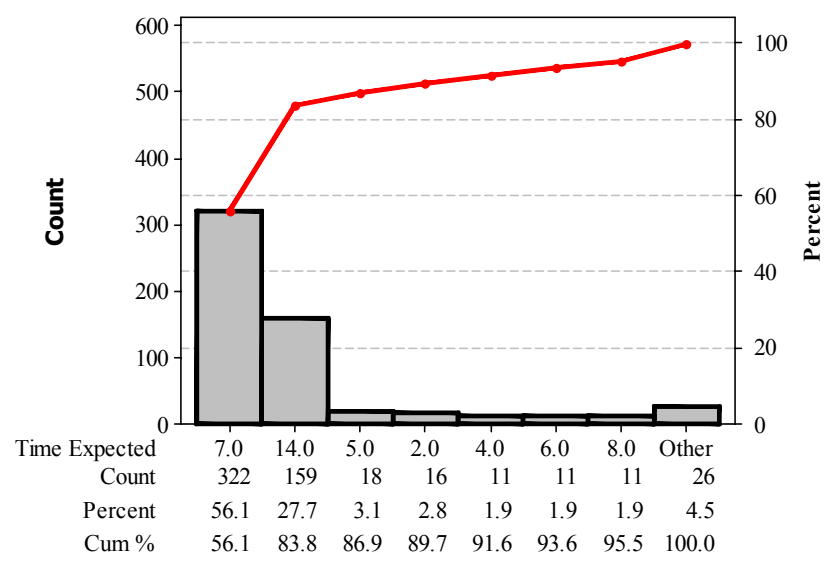

Figure 1: Pareto Chart of various "Time Expected"

The average actual cycle time for the reviwer to respond to the RFIs is longer than that of time expected for contactor to receive the response as following:

$\begin{array}{lrrrrr} & \text { Total } & & & & \\ \text { Variable } & \text { Count } & \text { Mean } & \text { StDev } & \text { Min } & \text { Max } \\ \text { Time Expected } & 574 & 8.572 & 3.885 & 0.500 & 37.0 \\ \text { Cycle Time } & 574 & 11.954 & 11.598 & 0.500 & 93.0\end{array}$

Table 1 summarizes the corresponding "on time" response rates to each "time expected." As mentioned previously, most of the RFIs were sent to the designer with tags 
of "time expected" in either 7 or 14 days, but their "on time" response rates were just $44 \%$ and $58 \%$ respectively. From this observation, we can conclude that expending due date is not a substantial solution to increase the on-time rate. Hence, we will look for another opportunity to improve the current RFI process - reducing the cycle time from a production system perspective.

Table 1: On-Time ratio corresponding different "Time Expected"

\begin{tabular}{ccccccccccccccccc}
\hline \multirow{2}{*}{$\begin{array}{c}\text { On- } \\
\text { Time }\end{array}$} & 0 & 1 & 2 & 3 & 4 & 5 & 6 & 7 & 8 & 10 & 11 & 13 & 14 & 16 & 37 & $\Sigma$ \\
\hline Yes & 2 & 2 & 6 & 6 & 3 & 8 & 4 & 143 & 5 & 1 & 1 & 0 & 92 & 0 & 1 & 274 \\
\hline No & 5 & 4 & 10 & 2 & 8 & 10 & 7 & 179 & 6 & 0 & 0 & 1 & 67 & 1 & 0 & 300 \\
\hline$\Sigma$ & 7 & 6 & 16 & 8 & 11 & 18 & 11 & 322 & 11 & 1 & 1 & 1 & 159 & 1 & 1 & 574 \\
\hline $\begin{array}{c}\text { \% of } \\
\text { On- } \\
\text { Time }\end{array}$ & 29 & 33 & 38 & 75 & 27 & 44 & 36 & 44 & 45 & 100 & 100 & 0 & 58 & 0 & 100 & 48 \\
\hline
\end{tabular}

\section{KEY POINTS INVOLVED IN CYCLE TIME REDUCTION STRATEGIES}

There are many ways to accomplish cycle time reduction from a production system perspective. The key methods for reducing cycle time are those that reduce mean flow time and flow time variance and there are three key points involved in cycle time reduction. (Hopp, Spearman, and Woodruff 1990)

\subsection{WIP and Flow Time}

Work-in-Process (WIP) and flow time are proportional to each other for a given level of throughput (TH). This means that causes of excessive cycle time can be determined by identifying locations with large inventories (WIP) (Hopp and Spearman 2000) In other words, we can accomplish the shorter cycle time by reducing WIP without making an effort to increase $\mathrm{TH}$.

\subsection{Inter-arrival Time and Process Time Variations}

As shown in the queuing delay equation, cycle time is related not only to the average of flow time but also to the variations of flow time and the utilization of server at workstation. Hence, even if the process time were stable, the waiting time in the queue will increase due to the variations resulting in inter-arrival times and process times of each entity that is supposed to be processed by the server(s) in the workstation. It is common occurrence that the larger the variation in the cycle time, the more safety time (as a buffer) is needed so as to absorb the impact of the variation. We can characterize the variation in both inter-arrival times and process times via the coefficient of variation in the queueing theory.
Hopp and Spearman (2000) established the classification of this variation into three groups - low, moderate and high variation - based on the magnitude of the CV value: LV (low variation) for CVs less than $0.75, \mathrm{MV}$ (moderate variation) for CVs between 0.75 and 1.33, and HV (high variation) for CVs greater than 1.33. In this research, we will investigate how variation affects the current RFI process performance in the context of queuing behavior and identify the key factors impacting the current process performance using the Design of Experiments

\subsection{Queuing and Waiting Times}

The two major components of flow time are queuing time and waiting time (Hopp and Spearman 2000). Since total run, setup, and move times typically make up only a fraction of the total cycle time, a large proportion is made up of waiting in queues, waiting for parts, and waiting to move (Hopp and Spearman 2000). Thus, it makes sense to focus effort on reducing the flow time associated with these components.

One of the well established GENERAL types of queuing models is the $\mathrm{G} / \mathrm{G} / 1$ model. Note that the first $\mathrm{G}$ denotes the type of distribution of inter-arrival time, the second $\mathrm{G}$ denotes the type of distribution of process times, and the last number " 1 " describes the number of servers at the workstation, respectively. The term "general" means that the G/G/1 model can take on any probability distribution as long as the inter-arrival times and process times are independent of one another. We can approximate the average waiting time of the $\mathrm{G} / \mathrm{G} / 1$ queue as follows (Hopp 2008):

$$
W_{q}^{G / G / 1} \approx\left(\frac{c_{a}{ }^{2}+c_{s}{ }^{2}}{2}\right)\left(\frac{\rho}{1-\rho}\right) \tau
$$

The above equation is known as Kingman's equation named after one of the first queueing researchers to propose it. Note that $C_{a}, C_{s}, \rho$, and $\tau$ denote the inter-arrival time Coefficient of Variation $(\mathrm{CV})$, the process time $\mathrm{CV}$, the utilization, and the average process time respectively. The expression shows that queuing delay is a multiple of the variations in the inter-arrival time and the process time, utilization and the average service time. Hence, by exploring the underlying causes affecting the values of these parameters, we can identify the factor(s) that cause waiting in a given queuing system (Hopp 2008)

\section{BATCH SIZES, INTER-ARRIVAL TIMES, AND CYCLE TIMES}

In order to understand the system behavior as accurate as possible, elements of key performance should be investigated rather than corporate-level summarial numbers (i.e. average). The raw data gathered was in date format so that 


\section{Chin and Russell}

the arithmetic difference between successive arrivals gives the inter-arrival times. For example, the inter-arrival time for RFI\#2 can be calculated by subtracting the date when RFI\#1 generated (2/7/2003) from the date when the RFI\#2 generated $(2 / 21 / 2003)$. The inter-arrival time is then 14 days. The inter-arrival times of entire RFIs data for the remainder of the RFI data were calculated in the same manner.

An interesting observation from the current process is the number of RFIs created on the same date. RFIs are not usually sent to the designer one at a time, but more often together in batches with different expected response times on the same date as shown in Table 2. On average, 4.63 RFIs were created and sent to the designer on the same date. The standard deviation of batch size was 4.00 and a median batch size was 4.00. This batching effect will be included in this study in order to see how the system performs differently. We will consider two different batch sizes - batch size of 1 and 4 which correspond to $5^{\text {th }}$ percentile (P5) and $50^{\text {th }}$ percentile (P50) of the batch size.

Table 2: Cycle Times and Inter-arrival Times

\begin{tabular}{ccccc}
\hline RFI \# & $\begin{array}{c}\text { Date } \\
\text { Created } \\
(1)\end{array}$ & $\begin{array}{c}\text { Date } \\
\text { Answered } \\
(2)\end{array}$ & $\begin{array}{c}\text { Cycle } \\
\text { Time } \\
(2)-(1)\end{array}$ & $\begin{array}{c}\text { Inter- } \\
\text { arrival } \\
\text { Time }\end{array}$ \\
\hline 1 & $2 / 7 / 2003$ & $2 / 12 / 2003$ & 5.00 & N/A \\
\hline 2 & $2 / 21 / 2003$ & $3 / 3 / 2003$ & 10.00 & 14.00 \\
\hline 3 & $2 / 27 / 2003$ & $3 / 5 / 2003$ & 6.00 & 6.00 \\
\hline 4 & $3 / 7 / 2003$ & $3 / 17 / 2003$ & 10.00 & 8.00 \\
\hline 5 & $3 / 7 / 2003$ & $3 / 17 / 2003$ & 10.00 & 0.00 \\
\hline 6 & $3 / 7 / 2003$ & $3 / 17 / 2003$ & 10.00 & 0.00 \\
\hline$\vdots$ & $\vdots$ & $\vdots$ & $\vdots$ & $\vdots$ \\
\hline \multicolumn{5}{c}{$\begin{array}{c}\text { RFI batch (created and arrived at the same } \\
\text { date at workstation) }\end{array}$} \\
\hline
\end{tabular}

The average inter-arrival time (of one or batched entities) was 1.90 days and its standard deviation was 1.74 (i.e. $\mathrm{CV}=1.74 / 1.90=0.92$, i.e. $\mathrm{MV}$ ). This can be interpreted to mean that one or a number of RFIs (batches) created by the contractor at the same date arrive at workstation (reviewer) every 1.90 days with a variation of 1.74 days.

An average cycle time of 11.95 days was observed with a standard deviation of 11.60 days $(\mathrm{CV}=11.60 / 11.95$ $=0.97$, i.e. $\mathrm{MV})$. This can be interpreted to mean that the servers take 11.95 days to complete a response to each RFI with a variation of 11.60 days. Table 3 summarizes each factor from the initial data analysis.

Table 3: Summary of each factor

\begin{tabular}{lcccccc}
\hline \multicolumn{1}{c}{ Factors } & Mean & Stdev & CV & P5 & P50 & P95 \\
\hline Inter-arrival Time & 1.90 & 1.74 & 0.92 & 1.00 & 1.00 & 4.90 \\
\hline Batch Size & 4.63 & 4.00 & 0.86 & 1.00 & 4.00 & 13.00 \\
\hline Cycle Time & 11.95 & 11.60 & 0.97 & 0.50 & 9.00 & 33.00 \\
\hline
\end{tabular}

\section{PROBABILITY DISTRIBUTION}

For each uncertain variable in a simulation, the possible values with a probability distribution should be defined in order to construct the most realistic model. The type of distribution depends on the conditions surrounding the variable. In order to determine the best fitting probability distributions, the Individual Distribution Identification - one of the facilities of Minitab software - was used.

\subsection{Inter-arrival Times}

In order to find the best fitting distribution for the interarrival times, the Minitab was run and Weibull was selected as the best fitting distribution given that the $\mathrm{AD}$ (Anderson-Darling) statistic of Weibull is the smallest number even though the corresponding P-value is less than 0.01 . The goodness of fit test was conducted based on a 95\% confidence interval. The Anderson-Darling statistic is a measure of how far the plot points fall from the bestfitting line in a probability plot. A smaller AndersonDarling statistic indicates that the distribution fits the data better (Mintab Inc. 2004). As a result the shape and scale parameters for the Weibull distribution were obtained as following:

$\begin{array}{lll}\text { Distribution } & \text { Shape } & \text { Scale } \\ \text { Weibull } & 1.35575 & 2.10697\end{array}$

\subsection{Cycle Times}

To find the best-fitting distribution for the cycle time, the same method used for finding the best-fitting inter-arrival time distribution was used. The result was similar to that for inter-arrival time and the Weibull- distribution was selected for the cycle time data as a best-fitting distribution.

\section{PROCESS CYCLE EFFICIENCY (PCE) AND PROCESS TIMES}

With the data gathered, we can only track the total cycle times which are the sum of waiting times and process times. For meaningful analysis in the context of queueing theory, measuring process times is critical. In the absence of this time data, it seems that no more ways exist to conduct the queueing related study.

The PCE is an important lean metric and the ratio of value-added time to total time required for producers to deliver goods to the customers (George, Rowlands, Price and Maxey 2005). It tells how fast the systems can response to the customer's demand. The larger the PCE value the leaner the system because the system has less fraction of non-value added times. However, we can also calculate the process efficiency using the number of fast RFI processed versus the total number of RFI processed (Muir 2006). We 


\section{Chin and Russell}

made an assumption here that 3 days are the cutoff between fast and slow process times.

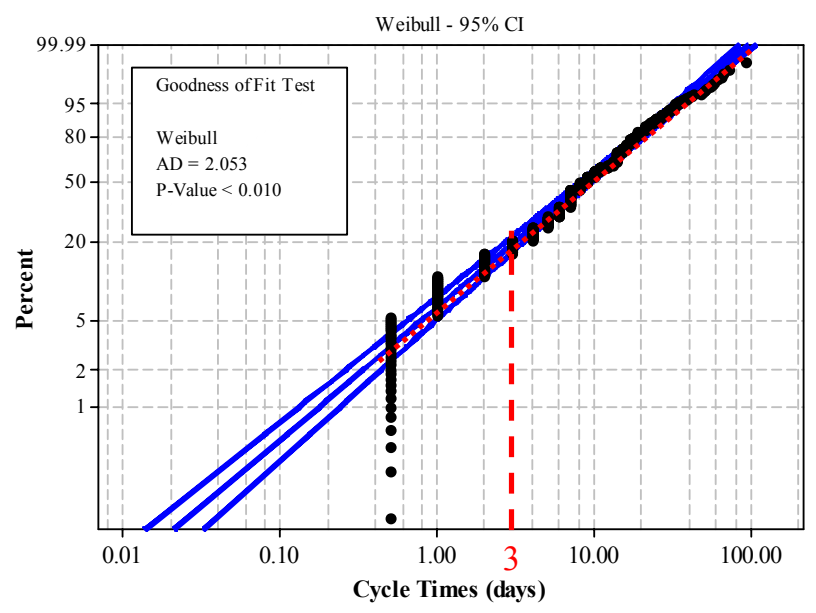

Figure 2: Probability Plot for RFI Cycle Times

Based on the probability plot as shown in Figure 2, the cycle time data can be separated into two groups (fast and slow processes). The dotted lines extrapolate the two cycle time components to an intersection to identify the fast process (left side) and the slow process (right side). Two dotted lines cross at about the $20^{\text {th }}$ percentile, meaning that about 20 percent of the RFIs are processed fast, while the remaining 80 percent are processed slowly. Following is the result of the descriptive statistics of cycle times (CT) for two categories.

Total

Var. fast/slow Cnt Percent Mean StDev Min Max

$\begin{array}{llllllll}\mathrm{CT} & \text { fast } & 120 & 0.209 & 1.54 & 0.92 & 0.5 & 3.0\end{array}$

$\begin{array}{lllllll}\text { slow } & 454 & 0.791 & 14.71 & 11.56 & 4.0 & 93.0\end{array}$

$\begin{array}{lllllll}\text { all } & 574 & 1.000 & 11.95 & 11.60 & 0.5 & 93.0\end{array}$

Then, the measure of process efficiency can be approximated by dividing the amount of time spent in the fast process by the total time spent in the process (Muir 2006).

$$
\text { Efficiency }=\frac{\left(\mathrm{p}_{\text {fast }}\right) \times\left(\mu_{\text {cycle, fast }}\right)}{\mu_{\text {cycle, total }}}
$$

Plugging the values observed into the above equation,

$$
\text { Efficiency }=\frac{\left(\frac{120}{574}\right) \times(1.54)}{11.95}=2.7 \%
$$

The results can be interpreted as when $20 \%(120 / 574)$ of the RFIs proceed through the process relatively quickly (processed within 3 days), the efficiency of the system is about $3 \%$. Hence, we can determine the average process time is about $3 \%$ of the average cycle time (i.e. 11.95 days x $0.03=0.36$ days). In order to examine different process performances, the same type of distribution but two different variations were considered as the process time parameter - i.e. average process times are 0.36 days but $\mathrm{CV}$ is either $0.32(\mathrm{LV})$ or $1.0(\mathrm{MV})$. Then, the main and interaction effects was observed.

\section{DESIGN OF EXPERIMENTS (DOE)}

It is hard to find the actual effects of many factors in the real situation because realistic scenarios can become very complex and nonlinear. Hence, it may become difficult to predict the effects of changes and to identify the most important factors in the process (Henderson 2006). However, the DOE enables one to explore the relationships between the key input process variables and the output performance characteristics and to identify or screen the important factors affecting output process performance. We constructed a simulation model using ARENA which is one of reliable commercial simulators and investigated the consequences of making changes to the input parameters using DOE. The simulation model constructed is fairly simple, but enough to understand the system behavior under different conditions.

\subsection{Levels of Inter-arrival Time and Process Time}

Two different levels were chosen on the assumption that each factor has same means but different variations. Figure 3 explains the different variations with the same means of process times. The red dotted line shows the low variation distribution for the process times with a mean of 0.36 days and a standard deviation of 0.12 days, which approximately follows the normal distribution - a bell-shaped curve. The black solid line represents the higher variation distribution with the same mean of 0.36 days. Directing an improvement effort toward making the process times more consistent (low $\mathrm{CV}$ ) would narrow the span on the process times (Muir 2006).

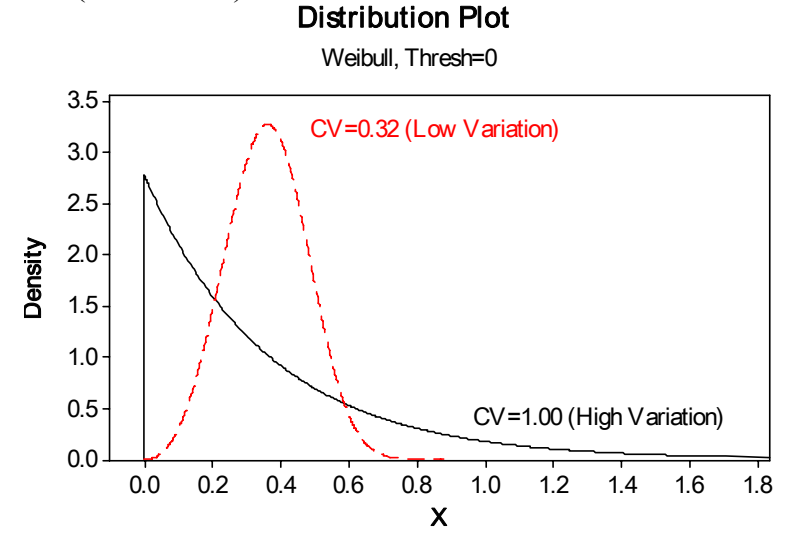

Figure 3: High and Low Variations 


\section{Chin and Russell}

Table 4 is the summary of simulation parameters for inter-arrival time and process time.

Table 4: Simulation Parameters

\begin{tabular}{|c|c|c|c|c|c|c|}
\hline Parameter & Type & Shape & Scale & Mean & StdDev & $\mathrm{CV}$ \\
\hline \multirow{2}{*}{$\begin{array}{l}\text { Inter- } \\
\text { arrival } \\
\text { Time }\end{array}$} & Weibull & 1.0 & 1.90 & \multirow[t]{2}{*}{1.90} & 1.90 & 1.00 \\
\hline & Weibull & 3.4 & 2.11 & & 0.62 & 0.32 \\
\hline \multirow{2}{*}{$\begin{array}{l}\text { Process } \\
\text { Time }\end{array}$} & Weibull & 1.0 & 0.36 & \multirow[t]{2}{*}{0.36} & 0.36 & 1.00 \\
\hline & Weibull & 3.4 & 0.40 & & 0.12 & 0.32 \\
\hline
\end{tabular}

Note that a Weibull distribution with a shape parameter of 1.0 is identical to an exponential distribution, while a Weibull distribution with a shape parameter of 3.4 is approximately normal (Muir 2006).

\subsection{Level of Batch Sizes}

In order to see how the process performs with different batch sizes, two different conditions were chosen - batch size 1 and 4, which correspond to P5 and P50 of the batch size observed in the current system respectively.

\subsection{Designing Experiments}

Now we can design experiments based on the factors and their levels as discussed previously. The objective of the study is to understand the queuing effects and relationship between three input factors (inter-arrival time, process time, and batch size) with two levels (low (-) and high (+)) so that experiments can be designed as the $2^{3}$ series. The results from an experiment can be used in determining effects of responses when factors move from low to high levels (or vice versa). Main effect of factor can be computed by applying the signs in the factor column to the corresponding responses, adding, and then dividing by $2^{\mathrm{k}-1}=$ 4 (Kelton 2000). For instance, the main effect of Factor A can be computed as:

$$
=(-\mathrm{R} 1-\mathrm{R} 2+\mathrm{R} 3+\mathrm{R} 4+\mathrm{R} 5-\mathrm{R} 6+\mathrm{R} 7-\mathrm{R} 8) / 4
$$

The main effects of other factors can be computed similarly. In addition, interaction effects among the factors can be also determined by multiplying the columns of the involved factors, apply the resulting signs to the corresponding responses, adding and then, dividing by $2^{\mathrm{k}-1}=4$ (Kelton 2000). For instance, the interaction between Factors $\mathrm{A}$ and $\mathrm{C}$ would be:

$$
=(+\mathrm{R} 1+\mathrm{R} 2+\mathrm{R} 3+\mathrm{R} 4-\mathrm{R} 5-\mathrm{R} 6-\mathrm{R} 7-\mathrm{R} 8) / 4
$$
Minitab.

\begin{tabular}{|c|c|c|c|c|c|c|c|c|}
\hline \multirow{2}{*}{$\begin{array}{l}\text { Std } \\
\text { Or- } \\
\text { der }\end{array}$} & \multirow{2}{*}{$\begin{array}{l}\text { Run } \\
\text { Or- } \\
\text { der }\end{array}$} & $\mathrm{A}$ & $\mathrm{B}$ & $\mathrm{C}$ & $\mathrm{A}$ & $\mathrm{B}$ & $\mathrm{C}$ & \multirow{2}{*}{$\begin{array}{c}\text { Re- } \\
\text { sponse }\end{array}$} \\
\hline & & \multicolumn{3}{|c|}{$\begin{array}{c}+- \\
\text { Notation }\end{array}$} & $\begin{array}{c}\text { Inter- } \\
\text { arrival } \\
\text { Time } \\
\text { CV }\end{array}$ & $\begin{array}{c}\text { Process } \\
\text { Time } \\
\text { CV }\end{array}$ & $\begin{array}{c}\text { Batch } \\
\text { Size }\end{array}$ & \\
\hline 1 & 1 & - & - & - & 0.32 & 0.32 & 1 & R1 \\
\hline 3 & 2 & - & + & - & 0.32 & 1.00 & 1 & R2 \\
\hline 6 & 3 & + & - & + & 1.00 & 0.32 & 4 & R3 \\
\hline 8 & 4 & + & + & + & 1.00 & 1.00 & 4 & $\mathrm{R} 4$ \\
\hline 4 & 5 & + & + & - & 1.00 & 1.00 & 1 & R5 \\
\hline 7 & 6 & - & + & + & 0.32 & 1.00 & 4 & R6 \\
\hline 2 & 7 & + & - & - & 1.00 & 0.32 & 1 & R7 \\
\hline 5 & 8 & - & - & + & 0.32 & 0.32 & 4 & $\mathrm{R} 8$ \\
\hline
\end{tabular}

Table 5: Matrix Designed for the Experiment

Note that the standard order in the above Table 5 indicates a structured pattern for each input factor while the run order provides a random sequence in which the experiment should be completed in order to arrive at statistically valid results (Minitab Inc 2004).

\section{SIMULATION MODEL}

The simulation model was constructed on the following assumptions and Figure 4 represented the simulation model built graphically.

- A total of 100 RFIs are fed into the model.

- Entity is released to the reviewer in a single unit (1 RFI) or in the form of a batch (4 RFIs) according to the different conditions for the simulation run.

- In the cases of batches, 4 RFIs are sent together to the reviewer (design team).

- The times required for batching are disregarded in the model.

- The number of server at workstation is one (i.e. one reviewer)

- Once the RFI(s) is received by the reviewer, the RFI(s) will be piled on the desk of the reviewer and subsequently picked up by the reviewer in FIFO (First-In First-Out) without consideration of any priority of the RFIs.

- It is assumed that there is no detractor in the process time (i.e. no setup times, breaks, or anything that extends the time required to complete processing of the entity).

- When the reviewer is busy, the rest of RFIs will be in queue and seized until the reviewer is available. In the simulation model, the "Seize" module was used to seize unit(s) of a resource (here in the model, one reviewer). When an entity (RFI) enters Seize module, it waits in a queue until a resource is available (Kelton, Sadowski, and Sadowski 2002) 


\section{Chin and Russell}

- No limit on the number of entities that can queue up at a workstation.

- Each simulation model is to run 1,000 times.

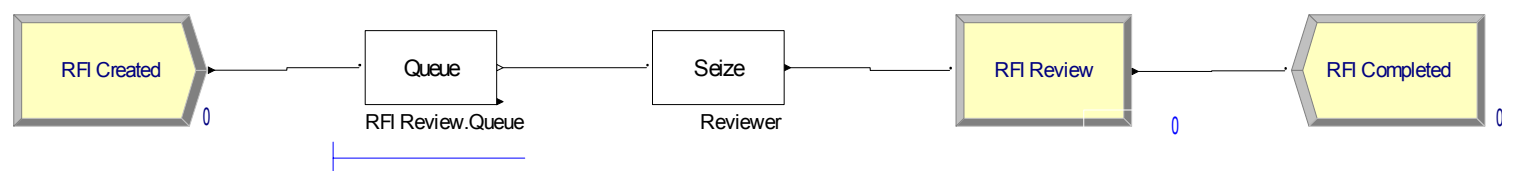

Figure 4: Simulation Model

\subsection{Simulation Results}

The parameters were chosen and the simulations were run in the run order created by the Minitab design of experiments. The same numbers of RFIs (100 RFIs) were processed in each of 8 scenarios, but their performances were quite different. Based on the results, Process Cycle Efficiency (PCE) was also computed. In estimating the PCE, we made an assumption that the process time $(\tau)$ only creates values but the waiting time $(\mathrm{Wq})$ does not add values at all. Table 6 is the summary of simulation results.

Table 6: Simulation Results

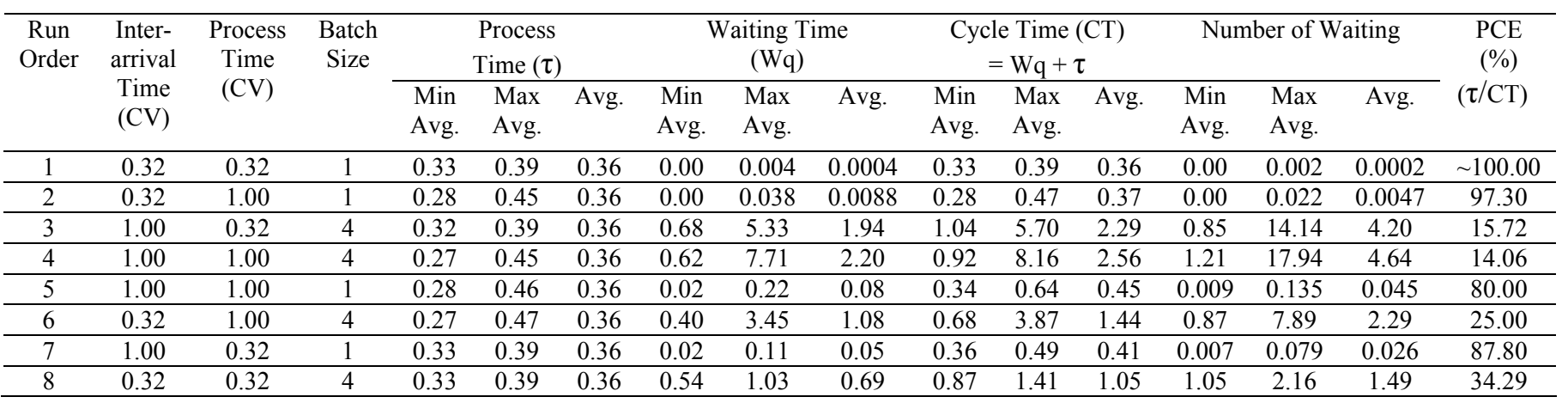

\subsection{Main Effects and Interaction Effects}

We can compute the main effect as described in the previous section. The following shows how to compute the main effect for the batch size (Factor C). The main effects of the other factors also can be computed in similar manners.

Batch size effect

$=(-\mathrm{R} 1-\mathrm{R} 2+\mathrm{R} 3+\mathrm{R} 4-\mathrm{R} 5+\mathrm{R} 6-\mathrm{R} 7+\mathrm{R} 8) / 4$

$=(-0.36-0.37+2.29+2.56-0.45+1.44-0.41+1.05) / 4$ $=5.75 / 4=1.437$

Figure 5 explains that on average increasing the coefficient of variation $(\mathrm{CV})$ of the inter-arrival time from 0.32 to 1.0 increases the cycle time of the RFI by 0.623 days (increasing from 0.805 days to 1.428 days). It also indicates that on average increasing the $\mathrm{CV}$ of the process time from 0.32 to 1.0 increases the cycle time of the RFI by 0.177 days (increasing from 1.028 days to 1.205 days). When the batch size increases from 1 to 4 , the cycle time of the RFI increases by 1.437 days (increasing from 0.398 days to 1.835 days). The main effect of inter-arrival time, the process time and the batch size are 0.623 days, 0.177 days, and 1.437 days respectively. Then, one can find the batch effect is greater than others.

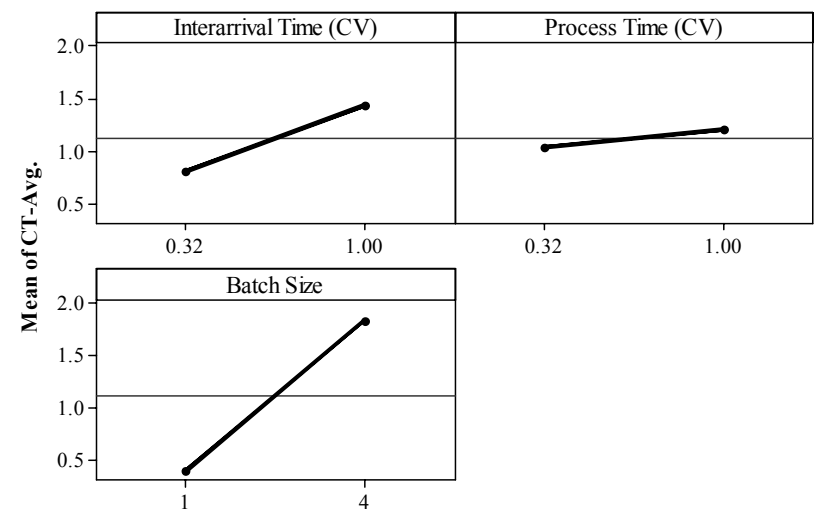

Figure 5: Main Effect Plot (data means) for Average Cycle Time

The primary goal of the DOE is to identify the "vital" few factors (key variables) that influence the output (response). The Minitab provides two graphs that help identify these influential factors - the Normal plot and the 


\section{Chin and Russell}

Pareto chart. These graphs allow one to compare the relative magnitude of the effects and evaluating their statistical significance. The Normal Probability Plot of the Effects and Pareto Chart of the individual and their combined effects indicate that the most effective factors to increase the cycle time are the batch size in the existing process. In the normal probability plot of the effects in Figure 6 , points that do not fall near the line usually signal important effects. Important effects are larger and further from the fitted line than unimportant effects. Unimportant effects tend to be smaller and centered around zero.

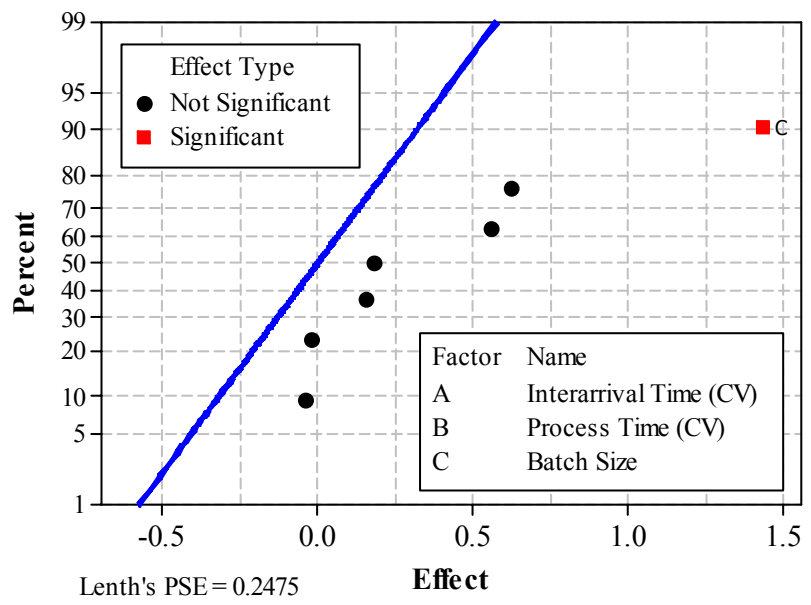

Figure 6: Normal Probability Plot of the Effect (Response is Average Cycle Time, Alpha $=.05$ )

The Pareto Chart in Figure 7 illustrates the effects in decreasing order of the absolute value of the effects. The reference line (red line) on the chart indicates which effects are significant. Again, for the RFI process, there is one significant effect - batch size.

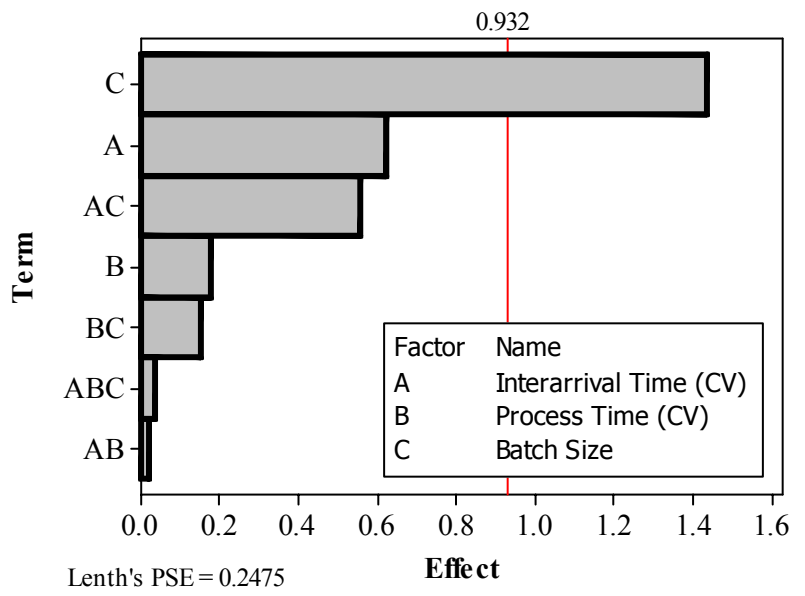

Figure 7: Pareto Chart of the Effects to Cycle Time Average (Response is Average Cycle Time, Alpha =.05)

When the effect of one factor depends on the level of another, the interaction plot in Figure 8 visually demon- strates the possible interactions. Parallel lines in an interaction plot indicate no interactions. The greater the difference in slope between the lines, the higher the degree of interaction. (Henderson 2006) We can compute each interaction effect as following:

- Interaction effect between inter-arrival time $\mathrm{CV}$ and process time $\mathrm{CV}$ (Factor $\mathrm{A}$ and $\mathrm{B}$ )

$=(+\mathrm{R} 1-\mathrm{R} 2-\mathrm{R} 3+\mathrm{R} 4+\mathrm{R} 5-\mathrm{R} 6-\mathrm{R} 7+\mathrm{R} 8) / 4$

$=(+0.36-0.37-2.29+2.56+0.45-1.44-0.41+$ $1.05) / 4=-0.09 / 4=-0.023$

- Interaction effect between process time $\mathrm{CV}$ and batch size (Factor B and C)

$=(+\mathrm{R} 1-\mathrm{R} 2-\mathrm{R} 3+\mathrm{R} 4-\mathrm{R} 5+\mathrm{R} 6+\mathrm{R} 7-\mathrm{R} 8) / 4$

$=(+0.36-0.37-2.29+2.56-0.45+1.44+0.41-$ $1.05) / 4=0.61 / 4=0.153$

- Interaction effect between inter-arrival time CV and batch size (Factor A and C)

$$
\begin{aligned}
& =(+\mathrm{R} 1+\mathrm{R} 2+\mathrm{R} 3+\mathrm{R} 4-\mathrm{R} 5-\mathrm{R} 6-\mathrm{R} 7-\mathrm{R} 8) / 4 \\
& =(+0.36+0.37+2.29+2.56-0.45-1.44-0.41- \\
& 1.05) / 4=2.23 / 4=0.558
\end{aligned}
$$

As a result, we can observe that interaction effect between inter-arrival time $\mathrm{CV}$ and batch size is relatively larger than other interaction effects.

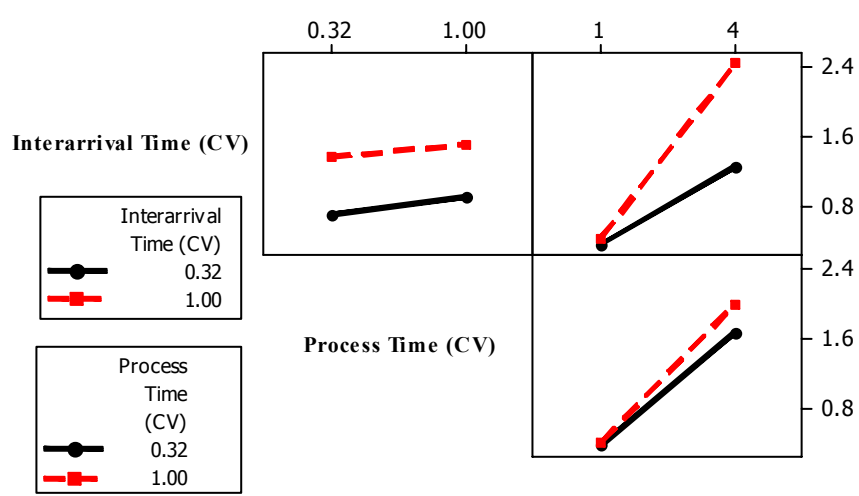

Batch Size

Figure 8: Interaction Plot (data means) for Cycle Time Average

A Cube plot in Figure 9 was also constructed to see the factors and combination of settings used in the design of experiments. We can see the low and high settings for the inter-arrival time $\mathrm{CV}(0.32$ and 1.00$)$, the process time CV (0.32 and1.00), and the batch size (1 and 4). It indicates that the combination of a low level of $\mathrm{CV}$ for both the inter-arrival time and the process time, and a low batch size (size 1) is the best scenario for the shortest cycle time when the average times for inter-arrival and process are same but their variations are different. 


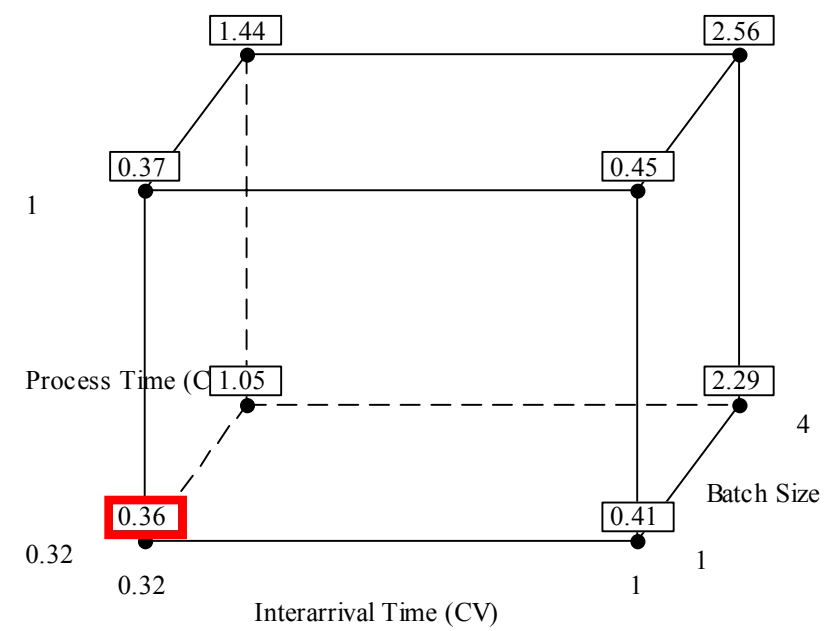

Figure 9: Cube Plot (data means) for Cycle Time Average

\subsection{Batching Effect}

As we observed, most significant cause of long cycle time was batch arrivals. This happens whenever jobs are batched together for delivery to a workstation. One might think that the variation is zero because entities batched arrive at a workstation simultaneously. However, looking at the inter-arrival times of each entity in the batch from the perspective of the individual RFIs, we will see different pictures (Hopp and Spearman 2000). In the RFI process, on average 4.63 RFIs are batched and delivered to the reviewer at the same time, but the reviews are done one at a time. From the observation, the inter-arrival time (i.e. time since the previous arrival) for the first RFI in the batch is 1.90 days. For the next 3.63 RFIs (4.63-1), it would be zero. hence, the mean time between arrivals $\left(t_{a}\right)$ is 0.41 days (1.9 days divided by 4.63 RFIs), and the variation of these times is,

$\sigma_{\mathrm{a}}{ }^{2}=\left[\frac{1}{4.63}(1.90)^{2}+\frac{3.63}{4.63}(0)^{2}\right]-t_{a}^{2}=\frac{1}{4.63}(1.90)^{2}-(0.41)^{2}=0.61$

The arrival Squared Coefficient of Variation (SCV) is therefore:

$$
C_{\mathrm{a}}^{2}=\frac{0.61}{(0.41)^{2}}=3.63
$$

As computed, the CV due to batching is quite large $(\sqrt{3.63}=1.91)$ compared to other sources of variation inter-arrival time CV (0.92) and process time CV (0.97)so that the batching effect increases the flow variation to a great extent and degrades the system performance resulting in longer cycle time (Hopp and Spearman 2000).

\section{CONCLUSION}

We have observed and discussed what the main and the interaction effects were in the current process. Now let's look at the current system (see Table 3). The CV of both the interaction and the cycle time are approximately one, and the median of the batch size is about four, which is one of the worst scenarios of the simulation.

The results from the design of experiments gave us an idea about where to start and how to maximize improvement efforts. In the RFI process, we found that the most significant factor to increase cycle times is the batch process. The large batch size causes large variation, increasing the number of waiting and the waiting times, consequently resulting in longer cycle times for RFI processing. In addition, we could observe different PCE levels given scenarios. The box plot of PCE vs. Batch size in Figure 10 indicates that a system with the batch size of one performs at a higher PCE level regardless of other factors.

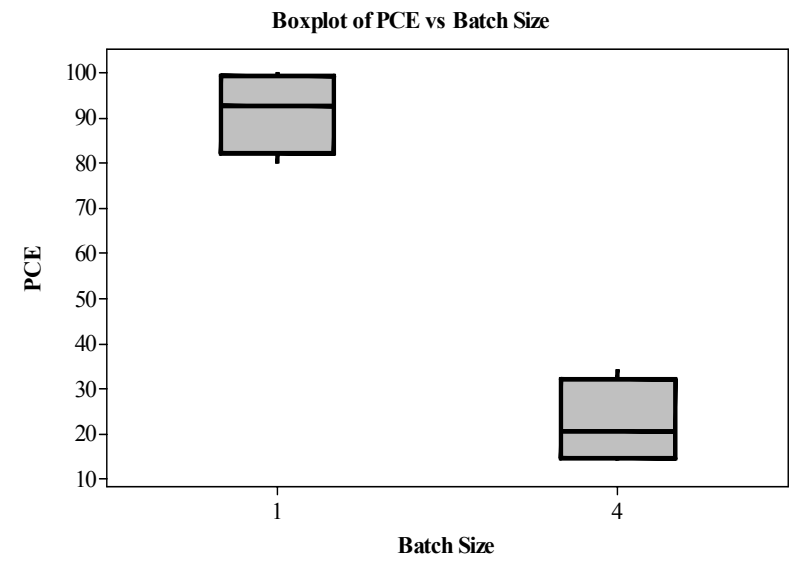

Figure 10: Boxplot of PCE (\%) vs. Batch Size

By knowing the problem area(s) as precise as possible, we can be directed to select the best options that are most likely to yield favorable results. There are numerous strategies to reduce variations. As mentioned previously, any improvement efforts toward making the factors more consistent (low $\mathrm{CV}$ ) would make process less variable but further details are not discussed here due to the limited space.

\section{REFERENCES}

George, M.L., D. Rowlands, M. Price and J. Maxey. 2005. Lean Six Sigma Pocket Tool Book. New York: McGraw-Hill.

Henderson, G.R. 2006. Six Sigma, Quality Improvement with MINITAB. John Wiley \& Sons, Ltd. UK. 
Hopp, W.J., M.L. Spearman and D.L. Woodruff. 1990. Practical Strategies for cycle time reduction. Manufacturing Review, Vol. 3, No. 2: 78-84.

Hopp, W.J. and M.L. Spearman. 2000. Factory Physics. New York: McGraw-Hill, $2^{\text {nd }}$ edition.

Hopp, W.J. 2008. Supply Chain Science. New York: McGraw-Hill.

Kelton, W.D. 2000. Experimental Design for Simulation. Proceedings of the 2000 Winter Simulation Conference, ed. J. A. Joines, R. R. Barton, K. Kang, and P. A. Fishwick, 32-38. Institute of Electrical and Electronics Engineers, Piscataway, New Jersey.

Kelton, W.D., R.P. Sadowski, D.A. Sadowski. 2002. Simulation with Arena. New York: McGraw-Hill, $2^{\text {nd }}$ edition.

Mintab Inc. 2004. Minitab ${ }^{\circledR}$ Help. State college, PA: Minitab Inc.

Muir, A. 2006. Lean Six Sigma Statistics-Calculating Process Efficiency in Transactional Projects. New York: McGraw-Hill.

Zack, J. G. 1999. Request for Information - Use, Abuse, and Control. Cost Engineering. Vol. 41, No. 9: 35-39.

\section{AUTHOR BIOGRAPHIES}

CHANG-SUN CHIN is Ph.D. candidate in the Department of Civil and Environmental Engineering at the University of Wisconsin, Madison. His research interest is in quality and performance improvement for construction operations using Lean and Six Sigma concepts. He can be reached at <mailto:chin2@wisc.edu>.

JEFFREY S. RUSSELL is Professor and Chair of the Department of Civil and Environmental Engineering at the University of Wisconsin, Madison. His research has centered around three areas: 1) construction contractor evaluation through such tools as prequalification; 2) computer automation and robotics; and 3) improvement strategies such as constructability and maintainability. He is a member of ASCE and his email and web address are <russelleengr.wisc.edu> and <http://www.engr.wisc.edu/cee/faculty/r ussell_jeffrey.html>. 